\title{
La gestion professionnelle des émotions et l'approche prothétique : un nouveau travail prescrit pour soigner les déments
}

The prosthetic approach to the professional management of emotions : a redefinition of prescribed work in the care of dementia patients

La gestión profesional de las emociones y el enfoque protésico : un nuevo trabajo prescrito para curar la demencia

\section{Michel Bigaouette}

\section{(2) OpenEdition}

\section{Journals}

\section{Édition électronique}

URL : http://journals.openedition.org/pistes/3806

DOI : 10.4000/pistes.3806

ISSN : 1481-9384

Éditeur

Les Amis de PISTES

Édition imprimée

Date de publication : 1 mai 2001

Référence électronique

Michel Bigaouette, «La gestion professionnelle des émotions et l'approche prothétique : un nouveau travail prescrit pour soigner les déments », Perspectives interdisciplinaires sur le travail et la santé [En ligne], 3-1 | 2001, mis en ligne le 01 mai 2001, consulté le 01 mai 2019. URL : http:// journals.openedition.org/pistes/3806 ; DOI : 10.4000/pistes.3806

Ce document a été généré automatiquement le 1 mai 2019.

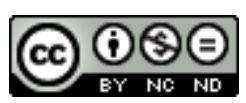

Pistes est mis à disposition selon les termes de la licence Creative Commons Attribution - Pas d'Utilisation Commerciale - Pas de Modification 4.0 International. 


\section{La gestion professionnelle des émotions et l'approche prothétique : un nouveau travail prescrit pour soigner les déments}

The prosthetic approach to the professional management of emotions : a redefinition of prescribed work in the care of dementia patients La gestión profesional de las emociones y el enfoque protésico : un nuevo trabajo prescrito para curar la demencia

\section{Michel Bigaouette}

\section{Introduction}

«Non, moi je ne veux plus me faire frapper! C'est trop dur le travail avec les déments. »

1 C'est avec ces mots qu'une préposée aux clients invite l'ergonome à intervenir dans son travail quotidien réalisé au sein d'un Centre d'hébergement et de soins de longue durée (CHSLD). Cette invitation est celle d'une rencontre avec les relations que les soignants entretiennent auprès de la clientèle de ces établissements. La clientèle des CHSLD est majoritairement composée de déments.

2 Comment se passe la vie de travail des soignants avec cette clientèle? Plutôt difficilement, car les comportements agressifs et perturbateurs (CAP) de la clientèle démente constituent autant d'énigmes à résoudre pour les soignants. Ces comportements surviennent souvent lors des contacts physiques intimes.

«Mais comment faire pour donner des soins quand on est obligé de toucher le client? Je ne peux pas faire autrement que de le toucher. Je n'ai pas le droit de le laisser dans sa merde ». C'est bien là le problème : comment dispenser ce soin avec cette clientèle particulière? 
3 À travers les mots des soignants, cet article nous permet de comprendre leur travail, en contact avec la clientèle démente lors des actes de soins. Nous verrons que la gestion de la vie émotionnelle de la clientèle constitue un élément fondamental du travail du soignant. Cette vie émotionnelle est au cœur du nouveau travail prescrit définie par l'approche "prothétique». Celle-ci vise notamment à solliciter les capacités résiduelles (psychologiques et physiques) de la clientèle démente. Elle agit comme une prothèse pour le client en déficit d'autonomie. Cette approche représente aussi pour le soignant une nouvelle façon de réaliser le travail. Telle une "prothèse », le soignant doit agir comme un accompagnateur auprès du client dément. L'utilisation professionnelle des émotions permet de jouer efficacement ce rôle d'accompagnateur.

\section{Accroissement de la clientèle démente dans les Centres d'hébergement et de soins de longue durée du Québec}

4 La population du Québec vieillit rapidement. L'impact de ce vieillissement commence à se faire sentir dans le réseau de la santé et des services sociaux. Au Québec, approximativement 48000 personnes âgées sont accueillies dans les CHSLD. Dans la vaste majorité des cas, cet hébergement est la conséquence directe d'une perte importante d'autonomie. Les déficits cognitifs associés à une démence constituent un aspect «fondamental» de la perte de l'autonomie. Le DSM 4 de l'association américaine de psychiatrie (1996) définit la démence sur la base des évidentes pertes de mémoire et la présence d'autres troubles. Les difficultés de reconnaissance (les agnosies), les difficultés à réaliser certains gestes (les apraxies) et les difficultés dans la communication verbale (les aphasies) sont des troubles fréquents. La maladie d'Alzheimer est certainement la forme de démence la plus connue du grand public.

5 La présence de la clientèle démente est très importante dans les CHSLD québécois. Dans plusieurs CHSLD, cette même clientèle constitue jusqu'à $50 \%$ de l'ensemble de celle hébergée. Selon toute vraisemblance, cette représentation pourrait encore s'accroître au cours des prochaines années si on se fie aux projections proposées dans la récente étude canadienne sur le vieillissement (1994). Selon les auteurs de cette étude, 8000 nouveaux cas de démence sont diagnostiqués annuellement au Canada dont environ 2000 cas au Québec. Il y aurait actuellement 75000 personnes âgées démentes au Québec. La moitié de ces dernières serait hébergée en CHSLD. D'ici 25 ans le nombre de déments aura plus que doublé, atteignant 156000 personnes au Québec. Cette réalité évolue silencieusement.

Les déficits cognitifs ont des conséquences directes sur le comportement de la clientèle. En 1996, une conférence de consensus organisée par l'International Psychogeriatric Association (Finkel et coll., 1996), a suggéré d'utiliser la dénomination: «signes et symptômes comportementaux et psychologiques" pour décrire les comportements agressifs et perturbateurs de la clientèle démente. Trois catégories existent: 1- les troubles comportementaux (ex: comportement d'errance, les comportements d'opposition et négativisme, les comportements intrusifs, l'apathie et l'indifférence et les conduites alimentaires inappropriées, les comportements stéréotypés, les conduites sexuelles inadéquates, l'agitation et les troubles du sommeil), 2- les troubles anxieux et de l'humeur (ex: l'anxiété, la dépression et l'humeur dépressive, l'irritabilité et les 
conduites agressives, la labilité émotionnelle, les réactions de catastrophe), 3- les signes "psychotiques» (ex : les idées délirantes, les hallucinations, les illusions).

7 La prévalence de ces signes et symptômes chez la clientèle est extrêmement élevée. Rovner et coll. (1986) situent la prévalence des troubles du comportement en soins prolongés entre 65 et $75 \%$. La proportion des troubles serait encore plus élevée, soit $90 \%$, dans les unités de soins accueillant spécifiquement une clientèle démente (Wagner et coll., 1995). L'étude menée par Bélanger et Dicaire (1993) dans deux établissements de la région de Québec situe la prévalence des troubles à $67 \%$. Nos propres études menées dans des CHSLD indiquent des résultats similaires.

\section{Satisfaire les besoins physiologiques, le travail actuel des soignants}

8 Actuellement, les services dispensés à la clientèle démente visent d'abord la satisfaction des besoins physiologiques. Il est généralement prévu que les infirmières et les infirmières auxiliaires s'occupent des besoins médicaux de la clientèle en collaboration avec les médecins (ex: préparation et distribution des médicaments). Quant aux préposées aux bénéficiaires, elles s'occupent en priorité des besoins de base qui assurent le confort et le bien-être physique de la clientèle. Concrètement, il s'agit des besoins alimentaires, des besoins de repos et de sommeil et des besoins d'hygiène.

9 Pendant son quart de travail, une préposée aux bénéficiaires a donc comme responsabilité de s'assurer que les besoins physiques de base de la clientèle sont comblés. Le tableau 1 présente l'exemple d'une journée de travail type d'une préposée.

Tableau 1. Activités menées par une préposée lors du quart de travail de jour

\begin{tabular}{|c|c|c|c|}
\hline Activités & $\begin{array}{l}\text { Début de } \\
\text { l'activité }\end{array}$ & $\begin{array}{l}\text { Durée totale } \\
\text { (en minutes) }\end{array}$ & $\begin{array}{l}\text { Durée moyenne } \\
\text { par client (en } \\
\text { minutes) }\end{array}$ \\
\hline Charger le chariot à lingerie & $7: 34: 10$ & $0: 42$ & \\
\hline Compléter la feuille de temps & $7: 35: 46$ & $1: 59$ & \\
\hline Installer les clients pour le déjeuner & $7: 36: 42$ & $25: 12$ & $2: 06$ \\
\hline Distribuer les cabarets et faire manger & $8: 02: 37$ & $24: 02$ & $3: 26$ \\
\hline Participer au rapport de l'infirmière & $8: 27: 58$ & $11: 33$ & \\
\hline $\begin{array}{l}\text { Enlever les cabarets et vérifier si le client a } \\
\text { fini de manger }\end{array}$ & $8: 39: 47$ & $5: 23$ & $1: 05$ \\
\hline Laver et vêtir & $8: 45: 45$ & $70: 42$ & $17: 41$ \\
\hline $\begin{array}{l}\text { Aller porter les poches de literie souillée et } \\
\text { les sacs de couches souillées à la chute à } \\
\text { linge et la chute à déchets }\end{array}$ & $10: 01: 49$ & $1: 27$ & \\
\hline
\end{tabular}




\begin{tabular}{|c|c|c|c|}
\hline Pause & $10: 03: 34$ & $19: 28$ & \\
\hline Laver et vêtir & $10: 24: 37$ & $55: 12$ & $13: 48$ \\
\hline Installer pour le dîner & $11: 21: 17$ & $3: 24$ & $1: 42$ \\
\hline Laver et vêtir & $11: 25: 34$ & $6: 25$ & $6: 25$ \\
\hline Installer pour le dîner & $11: 32: 03$ & $5: 58$ & $1: 59$ \\
\hline Préparer la distribution des cabarets & $11: 34: 48$ & $1: 37$ & \\
\hline Distribuer les cabarets et faire manger & $11: 35: 34$ & $1: 25$ & \\
\hline Installer pour le dîner & 52: 37: 11 & $1: 25$ & $1: 25$ \\
\hline Distribuer les cabarets et faire manger & $11: 38: 58$ & $12: 46$ & \\
\hline Installer pour la sieste (changer la culotte) & 02: 53: 11 & $28: 56$ & $2: 54$ \\
\hline $\begin{array}{l}\text { Aller porter les poches de literie souillée et } \\
\text { les sacs de couches souillées à la chute à } \\
\text { linge et la chute à déchets }\end{array}$ & $12: 23: 21$ & $1: 17$ & \\
\hline Dîner & $12: 25: 03$ & $50: 52$ & \\
\hline Changer la culotte & $13: 20: 39$ & $55: 45$ & $6: 58$ \\
\hline $\begin{array}{l}\text { Aller porter les poches de literie souillée et } \\
\text { les sacs de couches souillées à la chute à } \\
\text { linge et la chute à déchets }\end{array}$ & $14: 13: 52$ & $1: 21$ & \\
\hline Pause & $14: 15: 52$ & $22: 58$ & \\
\hline Changer de culotte & $14: 38: 55$ & $35: 50$ & $5: 58$ \\
\hline $\begin{array}{l}\text { Aller porter les poches de literie souillée et } \\
\text { les sacs de couches souillées à la chute à } \\
\text { linge et la chute à déchets }\end{array}$ & $15: 17: 41$ & $2: 08$ & \\
\hline Compléter le cahier de continence & $15: 19: 21$ & $0: 53$ & \\
\hline Fin de la journée & 18: 23: 15 & & \\
\hline
\end{tabular}

Données recueillies lors de l'observation des activités de travail d'une préposée suivie pendant un quart de travail complet (7h 30 à 15h 30).

10 Des données exploratoires recueillies en observant une préposée lors d'un quart de travail complet montrent que les activités visant à combler les soins d'hygiène accaparent $68,8 \% \mathrm{du}$ temps passé auprès des clients ( $4 \mathrm{~h} 13$ au total). Les soins d'hygiène impliquent des activités liées aux besoins d'élimination des selles et de l'urine (ex : changer la culotte de continence au lit, emmener le client sur la chaise d'aisance ou à la salle de toilette), aux besoins de propreté (ex: laver le client au lit ou au lavabo, enlever ou mettre un 
dentier à un client) et aux besoins d'esthétique (ex: parfumer, poudrer, etc..) et de prévention des blessures à la peau (ex : appliquer un onguent). Ces soins impliquent aussi la réalisation d'activités périphériques aux activités axées sur la satisfaction des besoins de base (ex: se déplacer, déplacer les clients dans le lit, du fauteuil au lit, éliminer les culottes et la literie souillée, compléter le cahier de continence afin d'identifier les clients qui souffrent de constipation etc..). Les activités visant à combler les soins d'hygiène sont réalisées pendant les trois quarts de travail.

11 Les préposées agissent ainsi pour de nombreuses raisons. Parmi ces raisons :

12 1) Elles doivent répondre aux demandes explicites verbalisées par les clients. Lors d'une étude sur les communications dans un CHSLD, nous avons observé que $91 \%$ des énoncés verbalisés par les clients non-aphasiques avaient pour objet la satisfaction d'un besoin de base. L'importance accordée aux soins physiques est illustrée dans l'extrait d'un journal d'observations d'actes de soins réalisé en soirée.

La mise au lit d'une cliente lucide

Client : Je voudrais avoir une jaquette bleue.

Préposée expérimentée : On va vous retourner, ce sera pas long, elle est partie voir s'il y avait une jaquette bleue.

Client : Ah oui ! Ah ben franchement, on n'est pas des bébés, hein, franchement.

C'est parce qu'elles sont plus chaudes que les autres.

Préposée expérimentée : OK, oui, oui, c'est vrai. C'est ça que vous m'avez dit hier.

Équipière : Bon ben, y'a aucune jaquette bleue sur tout l'étage. J'ai été voir dans les chariots des autres, dans la lingerie.

Client : Y'en a pas.

Équipière : Y’a une pénurie de jaquettes bleues.

Préposée expérimentée : Là, on la couche. Vous couche-t-on tout de suite?

Équipière : Non, moi je la coucherais plus tard (...)

Client : Je me couche tout de suite.

13 2) Elles doivent réguler avec des imprévus qui ont très souvent un impact sur le confort ou la sécurité du client. La gestion des imprévus oblige les préposées à s'engager encore plus profondément dans la réalisation de l'acte de soin physique.

Une cliente avec une démence modérée se cogne la tête lors d'une remontée à la tête du lit

Équipière : $O K$, on va la remonter après.

Client : Ben oui, faut pas l'oublier.

Équipière : La tête du lit est-elle baissée ? On dirait qu'il y a quelque chose de pris (imprévu no 1, une couverture est coincée dans la ridelle du lit).

Préposée expérimentée : Peut-être parce qu'elle a beaucoup d'oreillers.

Équipière : Allez-vous nous aider à vous remonter?

Client : Avez-vous enlevé l'oreiller ? L'avez-vous ôté ?

Préposée expérimentée : Non, non, vous avez les deux. On va juste la remonter un petit peu.

Équipière : $\mathrm{OK}$. Bon on va en enlever un (un oreiller) tout suite, ça va nous aider.

Client : C'est la plus grosse qu'on garde (..).

Équipière : Celle-là ici. Attends, je vais enlever mes affaires là. Bon.

Préposée expérimentée : $1,2,3$, go.

Client : Heu ! Hi ! Hi ! Hi ! (imprévu no 2, la tête de la client heurte la tête du lit)

Équipière : Vous êtes-vous cognée?

Préposée expérimentée : Êtes-vous correcte?

Équipière : Ben oui, est correcte, elle ne s'est pas cognée.

Client : Correct, correct.

Équipière : Y'a plus de peur que de mal. Y'avait une alèse dans son lit, c'est pour ça. 
14 3) Elles doivent travailler en équipe. Ce travail en équipe exige d'être planifié et coordonné. Les préposées doivent s'entendre entre elles sur leurs rôles respectifs et déterminer la manière de préparer l'activité, son déroulement et son suivi. Il faut donc harmoniser « les efforts ». Ceux-ci seront différents d'un client à l'autre car les soignants doivent réguler avec l'incontournable variabilité humaine (langue parlée, déficits sensoriels, niveau d'autonomie physique, etc..).

4) Elles doivent répondre aux exigences dictées par la culture hospitalière qui prévaut actuellement dans les CHSLD. Cette culture définit le client comme une personne «malade » et donc, dépendante dans les soins (Monat, 1999). Elle valorise une approche médicale du service à la clientèle. L'assise de cette approche repose sur la présence des facteurs biologiques spécifiques entraînant les dysfonctionnements d'un organe particulier. Avec les déments, ce sont les dommages au cerveau qui établissent les rapports de causalité entre l'étiologie, la neuropathologie et la symptomatologie. Ces rapports sont linéaires et directs. Le malade dément est qualifié d'organique, atteint d'une maladie souvent irréversible voire même incurable (Habib et coll., 1991).

\section{Les impacts de l'organisation actuelle des soins et des services aux clients déments : une réalité de travail alourdie}

Dans les CHSLD, directions, syndicats et employées s'entendent pour dire que l'organisation des soins et des services à la clientèle démente doit être revue. Pour les soignants, l'enjeu est l'alourdissement de la tâche. Les soignants croient qu'il existe un lien entre la survenue des CAP et l'alourdissement de la tâche. Plusieurs soignants ont beaucoup de difficultés à réguler avec les comportements agressifs et perturbateurs des clients déments.

17 Le témoignage recueilli auprès d'une préposée aux clients illustre une situation courante impliquant la dispensation des services visant la satisfaction des besoins physiologiques auprès de clients agressifs :

Ergonome : Quels types d'événements vous posent le plus de problèmes dans votre travail quotidien?

Préposée : Moi, je trouve que c'est quand tu arrives avec douceur et que le patient reste quand même agressif envers toi. C'est ça qui fait que tu as de la difficulté à « dealer » avec ça. Tu sais, quand le patient est perdu, qu'il a des médicaments mais c'est quoi qu'on doit faire? On se pose des questions. On se dit mais comment doiton procéder?

Ergonome : Vous ne le savez pas?

Préposée : Non

Ergonome: Au fond, vous ne savez pas comment arrêter un patient qui est agressif?

Préposée : Moi, je pense qu'on ne sait pas comment s'y prendre.

La recension des situations difficiles montre l'étendue et la variété des troubles du comportement ainsi que, les questions qu'adressent ces comportements aux soignants. Les questions les plus souvent entendues sont les suivantes:

"Que doit-on faire avec le client crieur? Comment donner un bain à un client qui s'y oppose physiquement? Quelle conduite adopter avec le client errant? Doit-on réprimer le comportement sexuel du client dément exigeant d'une cliente toute 
aussi démente des fellations à répétition ? Comment communiquer avec une cliente ayant perdue la capacité de communication verbale? ».

Ces questions révèlent le caractère "dépendant " de la clientèle. Cette dépendance alimente plusieurs croyances sur la perception du rôle de soignant. Si le client dément est dépendant, c'est donc qu'il est incapable de faire quoi que ce soit dans le quotidien. Il faut donc «faire à la place » du client, ce qui nécessite une organisation du travail valorisant un partage " égalitaire » entre les soignants des efforts à fournir dans le soin. Le partage du temps passé par les soignants auprès du client est un des enjeux centraux du partage des efforts.

Dans un sondage que nous avons réalisé en 1997 auprès de 1540 employés et cadres du réseau de la santé et des services sociaux, le « manque de temps » est considéré par $60 \%$ des répondants comme le premier facteur de pénibilité du travail (Bigaouette, 1997). Cette perception engage les soignants à faire des choix d'intervention au détriment d'une répartition du temps plus « équitable » où le partage du temps de travail serait déterminé par les besoins réels de chaque client. De plus, il semble exister un mécanisme de régulation du temps entre les différents quarts de travail. Plusieurs préposées affectées au quart de jour nous ont affirmé accélérer la cadence afin de ne pas donner de travail supplémentaire « aux filles du quart de soir».

Sous les pressions du temps de travail perçu comme disponible, les préposées observées font le choix d'enligner leur comportement sur la satisfaction des besoins de base de leurs clients. Ce comportement ne permet pas de satisfaire tous les besoins de leurs clients. Certains besoins tels que ceux visant la réalisation de soi (ex: initiative, création, développement de ses capacités, accomplissement, etc..), ceux visant l'estime de soi (ex : confiance en soi, savoir, liberté, indépendance, etc..) et la considération (ex : considération, être accepté, promotion, etc..) ou encore certains besoins sociaux (ex : être informé, s'exprimer, appartenance à un groupe, etc..) et de sécurité (ex : protection contre la discrimination, l'arbitraire, protection contre la perte de ce qu'on possède, etc..) ne sont pas visés directement dans les activités de soins.

Dans le travail réel, le comportement observé des préposées va se manifester par un activisme évident, à chaque étape de réalisation du soin. La participation du client est certes souhaitée (afin de maintenir son autonomie) mais, les faits montrent que cette participation est en réalité minime. Les préposées font généralement "à la place » du client, même si ce dernier est "lucide » et peut collaborer en fonction de ses capacités résiduelles. Dans le cas des clients déments, la participation est encore plus rarement sollicitée même si cela déplaît à ces derniers.

Le changement de culotte d'une cliente démente

Équipière : (elle tient les mains de la cliente) Mme X est mouillée. (...) La première fois, elle m'a donné un coup de pied.

client : (...) avec une belle police.

Équipière : Une belle police.

Client : Ouais.

Équipière : Vous, vous auriez fait une bonne police, ça j'en suis sûre.

Équipière : Ah ! Oups ! Y'a d'l'air, d'avoir des vapeurs (odeurs venant de la culotte). Juste vous tourner un petit peu. En! Y'en a des belles vapeurs.

Préposée expérimentée : (la préposée enlève la culotte souillée) Bougez pas. Mme $\mathrm{X}$.

Équipière : (la cliente tente de frapper l'équipière). Arrête de (...) Ouch! Mme X, là vous me faites mal.

Client : En? (...) 
Équipière : Ben oui, on a presque fini là.

Préposée expérimentée : Mme X, on va mettre votre culotte pis c'est fini après.

Équipière : (l'équipière tente de retenir la cliente qui veut sortir du lit toute nue).

Vous n'êtes pas pour vous promener toute nue sur la rue.

client: (l'équipière retient la cliente pour qu'elle reste au lit. La cliente frappe

l'équipière) Ayoye !

Préposée expérimentée : Attention pour pas qu'à parte, attention.

Client : (l'équipière retient solidement les mains de la cliente) Eh maudit !

Équipière : Attends, je vais être mieux les mains de même.

Préposée expérimentée : Tiens-y les mains, je vais m'arranger avec les fesses. OK.

Équipière : Bonne idée. Mme X, arrêtez.

Client : Lâche-moi.

Équipière : Lâche-moé.

Client : Lâche-moi.

Équipière : Lâche-moi.

Préposée expérimentée : $O K$, tournez un tit peu.

Équipière : Est orgueilleuse pareil, parce qu'à l'a vu que t'avais pas fini, c'est pour ça qu'à baissé la jaquette rien que ce bord là.

\section{La culture hospitalière est maintenant critiquée}

Ces dernières années, la culture de soin "hospitalière » existante dans les CHSLD a été largement critiquée. Les pressions populaires, les médias ont dénoncé les abus découlant de l'application de cette culture. La dénonciation émergente sur la maltraitance envers les aînés hébergés dans les CHSLD est un exemple concret de cette critique. Elle a été l'objet d'un plaidoyer à la Commission des droits de la personne du Québec en mars 2000. Pas moins de 108 mémoires ont été déposés et une cinquantaine d'organismes, associations et regroupements de personnes âgées ont été entendus. Le manque d'effectifs et de soins a été constaté notamment dans le mémoire déposé par l'Ordre des infirmières et infirmiers du Québec :

«Le déficit en soins infirmiers auquel (les personnes clients) doivent faire face n'est pas sans conséquence: il se traduit par une utilisation trop répandue des contraintes physiques et un manque de mesures de prévention des lésions de pression » (Le Devoir, 2000).

Le manque de soignants (infirmières, infirmières auxiliaires et préposées aux clients) serait un facteur important contribuant à la maltraitance. La satisfaction des besoins de base des clients hébergés ne serait pas comblée totalement mais partiellement (Le Devoir, 2000). Ces facteurs inciteraient plusieurs CHSLD et les associations d'employeurs à proposer une nouvelle approche de la dispensation des services.

L'approche "prothétique » émergente suggère cette nouvelle façon de dispenser les services et les soins à la clientèle. Elle redéfinit le rôle du client hébergé ainsi que celui du soignant. Ce dernier doit «accompagner» le client dément dans les activités de la vie quotidienne plutôt que "de faire à la place » du client. Selon cette approche, il faut solliciter les capacités résiduelles afin de les maintenir voire même contribuer à leur réadaptation.

Ainsi, le soignant compétent à gérer les comportements agressifs et perturbateurs serait celui qui aide le client à s'aider et non celui qui «fait à la place» de ce dernier. Ce qui signifie concrètement: observer, stimuler et initier les gestes du client lors de la réalisation des actes de soin. L'application de ces stratégies amène donc le soignant «à faire le moins possible ». 

travail des soignants. L'efficacité de cette approche repose sur la volonté affirmée des soignants de "transformer» leurs comportements encore inspirés de l'approche hospitalière. La transformation des comportements des soignants va dépendre notamment des avantages escomptés de l'approche prothétique dans la prévention des troubles du comportement.

\section{Les obstacles à l'implantation de l'approche prothétique}

Pour de nombreux soignants, ces avantages restent encore à démontrer. Ils doivent être tangibles, survenir dans un court délai, prévisibles et positifs pour les soignants et ce, dans la réalisation du travail réel. Par exemple, il faut que l'approche prothétique permette, via les plans d'intervention qui en découlent, de faciliter le changement de la culotte des clients déments. Cela est très important, car même dans un contexte prothétique, le contact intime et quotidien avec la clientèle démente reste présent.

Une fois identifiés, ces avantages doivent être planifiés et intégrés concrètement dans les plans d'intervention "prothétiques » individualisés proposés par les cliniciens pour éliminer les comportements agressifs et perturbateurs et favoriser l'adaptation des clients à leur milieu de vie.

Toutefois, afin que l'approche prothétique soit adoptée, il faudra que l'organisation des soins et des services reconnaisse l'efficience de la gestion professionnelle des émotions. Les psychologues comportementalistes ont beaucoup écrit sur ces émotions qui déterminent les comportements humains. Ainsi, ces derniers seraient déterminés par le contexte dans lequel se retrouve un individu et renforcés par les conséquences de ces comportements. Les émotions ressenties ou anticipées sont des conséquences de toute modification de comportement. La vie émotionnelle d'une personne dans un certain contexte serait modulée par ses croyances et ses idées. Tous ces éléments liés les uns aux autres situent les émotions au centre de cette chaîne des comportements humains (Cottraux, 1998 ; Auger, 1997).

31 Ce préambule sur la chaîne des comportements humains est nécessaire pour comprendre les avantages que pourrait procurer l'approche prothétique aux soignants à laquelle les employeurs et certains cliniciens demandent d'adhérer. Nous croyons que les soignants vont adhérer à l'approche prothétique seulement si cette dernière leur permet d'observer concrètement des améliorations dans la vie émotionnelle de leurs clients. clients et leurs actions professionnelles. Ils se perçoivent donc comme étant responsables des émotions ressenties par les clients. Pour ces derniers, le client connu comme agressif mais qui, lors d'un soin, ne frappe pas et collabore de manière harmonieuse est la conséquence directe d'un soin bien fait. Pour reprendre une expression d'Yves Gineste :

« Le soignant qui fait du bien se sent bien!» (Gineste et coll., 2001).

Cette référence à la vie émotionnelle des clients est un élément fondamental de la reconnaissance professionnelle à laquelle s'attendent les soignants dans le cadre de leurs activités de travail. $Y$ référer permet de bien comprendre l'activité réelle de travail du soignant en relation avec les déments. L'apprentissage du métier de soignant reposerait

Perspectives interdisciplinaires sur le travail et la santé, 3-1 | 2001 
sur les habiletés du soignant novice à gérer la question des émotions du client dans l'activité de travail.

Cette hypothèse découle d'observations des comportements de la clientèle. Naomi Feil a proposé une démarche visant à faire apprendre au soignant à se centrer sur la personne désorientée (1994). Selon cette auteure, le soignant professionnel est celui qui se met avec empathie à l'écoute du vécu de la personne âgée démente. L'approche développée par Feil repose sur un triple processus : accepter et prendre la personne là où elle en est ; accepter et reconnaître les émotions qu'elle ne contrôle plus et comprendre ainsi que valider les raison sous-jacentes aux émotions. La création d'un contexte favorable au ressenti d'émotions agréables serait donc la dimension principale de l'activité du soignant en relation avec le dément.

L'échec dans la mise en place d'un contexte affectif agréable a des conséquences émotionnelles importantes pour les soignants. En effet, les témoignages recueillis lors de formations sur la prévention des troubles de comportement en disent long sur les émotions désagréables qu'ils ressentent en regard de ces comportements. Les émotions les plus souvent décriées par les soignants sont la culpabilité, la peur, la colère, la peine ou un sentiment d'impuissance :

« Dès fois, je me dis, j'aurais dû faire autrement. C'est de ma faute » (culpabilité).

«Moi, je ne sais pas comment je vais être accueillie. Je sais que ce client-là est agressif. Coudon, il veut-tu me faire mal ? » (peur).

« Je suis souvent en « christ ». Je ne suis pas payée pour me faire frapper ». (colère).

«Ce client-là je ne le comprends pas. Un jour, il est calme et l'autre il frappe. Je ne

sais plus comment le prendre. Je me sens coupée de mes moyens, impuissante, qu'est-ce que je peux faire? As-tu des trucs à me donner?» (sentiment d'impuissance).

Les observations montrent que ces émotions désagréables desservent, dans les faits, les soignants car elles génèrent l'utilisation de stratégies défensives à l'endroit du client dément. La contention des mains, l'accélération des actes de soins sont des exemples fréquents. Malheureusement, ces stratégies ne font que renforcer les comportements agressifs et perturbateurs et par-delà génèrent des émotions désagréables pour les soignants.

La gestion professionnelle des émotions a fait l'objet de développements intéressants ces dernières années dans les CHSLD. Certaines stratégies de gestion émotionnelle ont permis de prévenir plusieurs comportements agressifs et perturbateurs. La gestion des réactions de catastrophe constitue un exemple concret. La réaction de catastrophe recouvre chez le client dément un état de détresse excessive qui se manifeste par des comportements émotionnels (ex: le client crie, se fâche, pleure) et moteurs (ex : agitation, lancer des objets, frapper, etc..) dramatiques ou violents. La fréquence de ces comportements est très importante. Par exemple, dans un CHSLD de Montréal, nous avons observé que $25 \%$ des 181 clients hébergés ont des réactions catastrophiques. Ces comportements surviennent plus fréquemment lors des toilettes partielles, du bain ou de l'habillage/ déshabillage. De manière moins spectaculaire, la réaction de catastrophe peut se manifester par de l'apathie, le retrait, le déni ou l'accusation (Ylieff, 2000). La fréquence des réactions de catastrophe est liée à la gravité du déficit cognitif.

Les neuropsychologues Taillefer et Geneau (1999) ont modélisé l'utilisation d'une stratégie de diversion pour prévenir les réactions de catastrophe. La diversion vise à centrer l'attention du client dément sur un événement de vie ou un objet ayant une 
charge affective positive pour ce dernier. L'attention du client est donc référée à des événements émotionnels de sa vie passée ou encore à des habiletés développées antérieurement (ex: faire égrener un chapelet à un client dément ayant eu une vie spirituelle importante dans le passé). Cette stratégie est très efficace chez les déments de type Alzheimer et d'autres catégories de déments. Le soignant dispose donc d'une stratégie de gestion de la vie émotionnelle du client dément. Cette façon de faire puise dans la vie passée du client afin de faire revivre chez ce dernier des émotions agréables.

L'utilisation des stratégies de diversion est un des trucs du métier bien connu des soignants expérimentés qui « intuitivement » en avaient déjà découvert l'efficacité :

Préposée : Des fois, moi j'y donne des débarbouillettes pour qu'y s'amuse au lavabo, le temps que tu le laves.

Infirmière : $\mathrm{Ah}, \mathrm{OK}$.

Préposée : Ca va y changer les idées. Tu le couches sur le côté face à la fenêtre pis tu montes pas la ridelle, hein!

Infirmière : Non, $\mathrm{OK}$.

Gineste et Marescotti (Gineste et coll., 2001) ont aussi proposé des stratégies de gestion émotionnelle efficaces reposant sur une philosophie de soin novatrice et très pratique. L'apport original de ces derniers concerne notamment l'utilisation du toucher professionnel affectif. Ce toucher professionnel vise un maximum de douceur par l'évitement de prises en pince lors du déplacement d'un membre. Il vise aussi à maintenir un contact continu avec la peau du client lors de la toilette. Le soignant qui utilise le toucher professionnel affectif parle au cerveau émotionnel du client à savoir le cortex limbique. Gineste reprend ici l'idée développée par le neuropsychologue américain Damasio qui, dans son livre "L'erreur de Descartes" (1994), démontre que la vie émotionnelle est notamment crée à partir des informations provenant des cinq sens.

La gestion émotionnelle est un fondement de l'approche à la clientèle démente. Elle est utilisée dans le contexte actuel supporté par la culture hospitalière. On la retrouve dans le discours des soignantes. Celui-ci met l'emphase sur la dimension affective comme moyen de gérer les CAP des clients. Par exemple, avec un client reconnu comme ayant un potentiel agressif élevé, elle utilise une stratégie visant à saluer le client et lui sourire le plus souvent possible dans une journée :

«J'essaie de lui dire bonjour pour qu'en partant, il m'en veuille pas. Donc je vais le voir, je lui dis bonjour à chaque fois que je passe. Je lui dis bonjour, «êtes-vous de bonne humeur? » Là il me fait signe. Des fois, il ne me regarde pas et ne me parle pas. Des fois, il va me dire oui. Mais des fois il va dire : «va-t'en là-bas ». Mais à chaque fois que je passe, je le regarde et je lui fais un sourire, parce que je le sais que dans le fond que ça lui fait du bien. Comme c'est moi qui le couche, c'est important ».

43 Avec une cliente reconnue comme étant agressive, elle va aussi être dans le registre affectif et accepter des marques d'affection de sa part:

«Hier, quand on est allé la coucher, elle a voulu me donner un bec. Je me suis penchée, elle m'a donné un bec ».

Pour la préposée expérimentée, être dans le registre affectif est essentiel. Les clients sont des personnes âgées à qui on doit le respect :

«Moi, je me dis, écoute là, c'est pas des boîtes de conserve, on travaille avec des personnes âgées. Je pense qu'il faut quand même que tu te mettes dans leur peau. S'il fallait que ce soit maman qui soit comme ça, j'aimerais pas ça moi que la fille 
fasse n'importe quoi. Ce serait ma mère, ce serait ma belle-mère, ce serait ma grand-mère, ça c'est des choses comme ça, tu vas te mettre dans la peau. Fais que je me dis " coudon, peut-être qu'elle va être contente à soir de se coucher plus tard", et peut-être que l'autre va être contente de se coucher plus de bonne heure, c'est ça ».

Pour établir la relation avec le client dément, elle va tenter de créer un contexte de travail affectif propice à la réalisation d'un soin bien fait :

« Entre toi et moi, quand je suis avec la patiente, ça peut très bien être Mme X, mais ça peut aussi bien pour Mme $Y$, je vais lui dire, «viens-t'en ma belle Alice, viens mon petit cœur d'amour ». Et là je vais la ramasser, je vais la coller pour qu'elle sache que je lui veux pas de mal, Si elle se sent en confiance avec moi à ce moment, je vais faire des farces avec elle. Je vais jaser, mais par contre, le lendemain si je la sens agressive et que je suis plus fatiguée, bien je m'arrêterai à essayer de la forcer. Je vais lui dire, ben écoute Mamie là, je suis fatiguée à soir-là, donne-moi un petit coup de main, veux-tu?»

L'enjeu de l'utilisation d'une stratégie affective est d'augmenter la collaboration du client et d'éviter les comportements agressifs et perturbateurs, manifestation de la peur :

«Surtout les Alzheimer, comme M. X. Mettons que tu veux le coucher ou que tu veux le changer, ben là je vais lui dire « tu viens-tu mon grand-papa d'amour, viens on va aller prendre une belle marche. Eh que tu as l'air fatigué Papi. Viens, on va te mettre en jaquette et on va te laver ». Souvent la direction nous dit qu'on devrait pas fonctionner dans ce sens-là, parce que tu dois pas leur dire «tu». Mais, il me semble que pour moi, c'est beaucoup plus facile d'arriver pis de dire «Viens grandpapa d'amour je vais faire ta toilette, et après ça on va se mettre en jaquette " ".

Pour cette préposée, l'utilisation d'une stratégie affective permet de dépasser la «barrière » de la sacro-sainte distance professionnelle. L'affection lui permet de se rapprocher du client, de cette personne humaine qui est avec elle.

La préposée insiste aussi sur l'harmonisation des émotions des soignants qui doivent faire équipe :

Préposée : Et puis y'a une chose qui est très, mais très très importante, c'est la préposée avec qui tu travailles. Si tu sens que ta préposée est nerveuse, veut veut pas la patiente va être nerveuse, pis toi tu vas l'être nerveuse.

Ergonome : Donc, comme autre condition, tu me parles d'être calme?

Préposée: Oui, parce que, admettons je travaille avec une personne qui est excessivement nerveuse, moi je vais essayer de lui communiquer quelque chose pour la calmer. Mais, cela peut ne pas être évident pour la préposée qui est avec moi. Je vais alors lui dire, «Bon, fais-toi en pas avec ça, on finit à $11 \mathrm{~h} 30$, c'est pas plus grave que ça, si on n'a pas fini la job». Si on n'a pas fait tous les clients, c'est pas grave. Moi, je leur dit toujours ça mais c'est pas écrit dans les livres ».

Finalement, l'objectif avoué de l'utilisation d'une stratégie affective est la confiance du client :

Ergonome : À quel genre de relation crois-tu que le client s'attend lorsque tu vas lui donner des soins d'hygiène?

Préposée : La confiance, la première chose, la confiance.

Ergonome : Il faut que la confiance s'installe?

Préposée : Oui, pour moi oui.

Ergonome : C'est quoi ça la confiance, ça se manifeste comment?

Préposée : Bon mettons. Je te donne un exemple, mettons, Mme X qui est très très agressive, je vais lui demander: $\mathrm{Tu}$ veux un petit mot vulgaire? Je vais tenter d'atténuer son comportement par l'humour. Ou encore, je vais dire : « Viens grandmaman, on va te changer " pour tenter de rentrer dans sa tête. Je vais essayer de m'intégrer à elle pour essayer d'avoir de la confiance. Faut pas qu'elle ait peur. Il 
faut qu'elle comprenne ce que je veux d'elle. Pour moi, c'est ça. Aussitôt qu'il va y avoir un client qui va être agressif, la première chose que je vais essayer c'est qu'elle me fasse confiance.

\section{La gestion des troubles de comportement de la clientèle démente par les soignants : l'accompagnement utile de l'ergonome}

La question des troubles du comportement de la clientèle démente est vaste et concerne plusieurs champs d'expertise. L'ergonome peut être très utile aux soignants pour qui la question des troubles de comportement est d'abord affaire d'efficience. Le regard porté par l'ergonome sur l'activité de travail peut concilier les besoins de prévention des troubles exprimés par les soignants.

1. Face aux diverses réalités de soins, les soignants recherchent fréquemment "des trucs" pour prévenir ces troubles. Ils veulent adapter leur conduite correctement. Ils réfèrent en premier lieu aux cliniciens afin de connaître les meilleures stratégies d'approche. Toutefois, l'application concrète des stratégies prescrites par les cliniciens n'est pas toujours un succès en situation de travail réel. Les conditions d'application liées à l'organisation du travail, l'environnement, les équipements ou encore les soignants eux-mêmes, sont autant de facteurs déterminants du succès de l'application de ces stratégies. Sur ces questions, l'ergonome peut apporter «des trucs» (ex: le choix d'équipements, l'aménagement de l'espace, etc..).

2. La participation de l'ergonome est aussi utile dans l'analyse des situations à risque pour la santé des soignants. Ces problèmes de santé sont fréquents et importants. Globalement, les statistiques de la CSST montrent que l'incidence des lésions professionnelles est plus élevée dans les CHSLD que dans toutes les autres catégories d'établissement du réseau de la santé et des services sociaux. Cette situation existe depuis au moins 1983. En 1995, 15 \% des soignants employés dans les CHSLD ont été indemnisés à la suite d'un accident de travail ou d'une maladie professionnelle. Ce taux est trois fois plus élevé que dans les centres hospitaliers psychiatriques qui accueillent aussi une clientèle présentant un problème de santé mentale ou un trouble organique. Par ailleurs, les statistiques sur l'assurance-salaire indiquent que les soignants des CHSLD sont les plus à risque de s'absenter de leur travail à la suite d'une maladie. En 1996, le taux d'absence (mesuré à partir des montants payés en assurancesalaire et des heures travaillées) était plus élevé dans les CHSLD que dans toutes les autres catégories d'établissement du réseau de la santé et des services sociaux. Autre élément intéressant, ce sont les employés âgés de 50 ans et plus qui sont le plus à risque de s'absenter du travail à la suite d'un accident du travail ou d'une maladie. L'hypothèse serait donc que l'exposition répétée à la clientèle démente aurait un impact significatif sur les soignants vieillissants.

3. L'ergonome peut aussi collaborer à l'identification des stratégies gagnantes pour améliorer le travail des soignants. Ces stratégies concernent le choix de la toilette la plus adéquate pour le client et pour le soignant. Le client doit-il être lavé au lit, debout au lavabo, à la baignoire ou à la douche ? Ces stratégies gagnantes visent aussi d'autres activités telles que l'alimentation du client (ex: Faut-il privilégier les aliments solides ou liquides? Doit-on asseoir et attacher le client agité lors des repas ou plutôt le laisser déambuler avec un sandwich à la main ? Où faut-il asseoir les clients pour éviter les conflits entre des clients "perturbateurs»?) ou la participation du client dans des activités de loisir (ex: Vaut-il mieux intégrer le client dans des activités de groupe ou dans des activités individuelles ?, etc..). 
4. L'ergonome peut aussi apporter une contribution par rapport à la gestion des émotions. Il peut assister les directions patronales et syndicales des CHSLD à l'implantation de l'approche prothétique.

5. Cette collaboration peut se faire :

- par l'étude des modes de gestion des émotions. Celles-ci peuvent susciter des stratégies défensives dysfonctionnelles par rapport à ce que l'on vise: donner des soins avec un minimum de risque

- par la mise en valeur de stratégies qu'utilisent déjà les soignants dans leur pratique quotidienne.

\section{Conclusion}

Dans cet article, nous avons montré que la réalisation des activités des soins menées par les soignants en contact avec la clientèle démente n'était pas simple. La survenue des comportements agressifs et perturbateurs de la clientèle représente des dangers pour la santé et la sécurité du travail des soignants et de la clientèle elle-même. La prévention de tels comportements passe par une modification dans la réalisation des activités de travail. Ce travail mené auprès des déments est multidimensionnel. Pour le mener à bien, il est nécessaire de répondre à certaines conditions. La reconnaissance de la pertinence de la gestion professionnelle des émotions est une condition fondamentale de la prévention des CAP.

Cette reconnaissance répond à une réalité complexe constituée autour d'un enjeu important: la difficulté pour les soignants d'adopter de nouvelles stratégies d'intervention. Cette difficulté peut être surmontée par l'implantation d'une approche prothétique. Cette dernière implique un nouveau travail prescrit où chaque soignant devient un accompagnateur dans le maintien, voire même le rétablissement des capacités résiduelles du client dément plutôt qu'un simple dispensateur de soins de base. Sur la question de l'implantation, l'ergonome a un rôle essentiel soit celui d'améliorer les conditions du travail du soignant.

L'intervention de l'ergonome peut donc contribuer à faire ressortir la valeur exceptionnelle $\mathrm{du}$ travail des soignants. Les CHSLD représentent un terrain extraordinaire pour l'ergonome qui veut comprendre les liens qui unissent le monde des émotions aux gestes et paroles professionnels. Et c'est bien là le défi : pour reprendre l'expression d'Albert Jacquard, les interventions de l'ergonome peuvent contribuer à ajouter de «l'humanitude» dans le travail. Il s'agit ainsi de favoriser l'adoption de stratégies d'intervention rigoureuses reposant sur la reconnaissance du travail bien fait, l'absence de répétition de situations frustrantes lors de la réalisation des activités. Ces stratégies doivent de plus faire activement appel à l'intelligence émotionnelle des soignants. Ajouter de "l'humanitude», c'est le travail réel des soignants envers les déments et nous, humbles mortels, qui vieillissons aussi. 


\section{BIBLIOGRAPHIE}

Association américaine de psychiatrie (1996). DSM-IV, Manuel diagnostique et statistique des troubles mentaux. Masson.

Auger, L. (1997). 21 jours pour apprendre à travailler sans vous détruire. Collection microthérapie, Centre interdisciplinaire de Montréal.

Bélanger, M., Dicaire, L. (1993). L'agitation en établissement d'hébergement. La Gérontoise, 4, 2, 12-19.

Bigaouette, M. (1997). Et la santé comment ça va ? Objectif Prévention, 20, 4, 16-19.

Bigaouette, M. (1997). Sondage - Et la santé, comment ça va ? Les facteurs déterminants de la santé. Objectif Prévention, 20, 5, 3-8.

Canadian medical association journa (1994). Canadian Study on Health and Aging Working Group. Study Methods and Prevalence of Dementia, 150, 899-913.

Cottraux, J. (1998). Les thérapies comportementales et cognitives. 3e édition, Masson.

Damasio, A (1994). L'erreur de Descartes. Édition Odile Jacob.

Dufour, V. (200). Soins de longue durée : les vieillards maltraités. Le Devoir, 9.

Feil, N. (1994). Validation, pour une vieillesse pleine de sagesse ! $2^{\mathrm{e}}$ édition, Éditions Pradel.

Finkel, S.I., Costa Sylvia, J., Cohen, G., Miller, S., Sartoriaus, N. (1996). Behavioral and Psychological Signs and Symptoms of Dementia : A consensus Statement on Current Knowledge and Implications for Research and Treatment. International Psychogeriatrics, 8, suppl, 497-500.

Gineste, Y., Marescotti, R. (2001). Philosophie des soins : Qu'est-ce qu'être soignant? Communication et Études Corporelles.

Habib, M., Joanette, Y., Puel, M. (1991). Démences et syndromes démentiels. Approche neuropsychologique. Masson.

Monay, A. (1999). Répondre aux besoins spécifiques de la clientèle atteinte de déficits cognitifs : un défi réalisable. Cahier de formation.

Rovner, B.W, Kafonck, S., Filipp, L., Lucas, M.J., Folstein, M.F. (1986). Prevalence of Mental Illness in Community Nursing Home. American Journal of Psychiatry, 143, 11, 1446-1449.

Taillefer, D., Geneau, D. (1999). Stratégies de diversion dans la gestion des réactions catastrophiques chez la personne âgée atteinte de la maladie d'Alzheimer lors d'actes de soins critiques : un cadre théorique et pratique. Centre de consultation et de formation en psychogériatrie. Wagner, A.W., Trei, L., Orr-Rainey, N. (1995). Behavior Problems of Residents with Dementia in Special Care units. Alzheimer Disease and Associate Disorders, 9, 3, 121-127.

Ylieff, M. (2000). Prise en charge et accompagnement de la personne démente. Les cahiers du troisième âge, éditions Kluwer. 


\section{RÉSUMÉS}

Les soignants qui travaillent auprès des déments font face à d'importantes difficultés. Leurs activités de travail concernent notamment la prévention des comportements agressifs et perturbateurs chez la clientèle démente. Les stratégies utilisées pour prévenir ces conduites représentent un des enjeux fondamentaux de l'activité de travail. Elles réfèrent à la gestion professionnelle des émotions ressenties non pas seulement par les clients lors des actes de soin mais aussi à celles éprouvées par les soignants eux-mêmes.

L'intervention de l'ergonome auprès des soignants œuvrant dans les Centres d'hébergement et de soins de longue durée vise notamment à documenter ces stratégies émotionnelles utilisées par les soignants. Elle vise également à supporter les directions patronales, syndicales et les employés de ces établissements dans l'implantation de modèles d'organisation de service et de soins plus efficients. L'approche " prothétique » constitue un exemple. Cette démarche redéfinit le travail prescrit du soignant. Elle vise une meilleure satisfaction des besoins fondamentaux de la clientèle démente.

Caregivers who work with dementia patients face some significant difficulties. Among other things, their tasks involve preventing aggressive and disruptive behaviour among their patients. The strategies they use to do this are one of the fundamental issues in their work activity, and include professional management of the emotions experienced by patients during care activities, as well as those experienced by the caregivers themselves.

An ergonomist worked with caregivers in residential and long-term care centres, mainly in order to document the emotion-related strategies used by caregivers, but also to support employers, union authorities and employees in the centres as they implemented more efficient care and service organization models. The "prosthetic " approach is an example of the ergonomist's contribution. This approach redefines the caregiver's prescribed work, in order to satisfy the basic needs of dementia patients in a more effective way.

Los cuidadores que trabajan con las personas dementes enfrentan muchas dificultades. Sus actividades de trabajo a menudo consisten en la prevención de comportamientos agresivos y perturbadores con esta clientela. Las estrategias utilizadas para prevenir estas conductas representan uno de los desafíos fundamentales de la actividad profesional. Se refieren a la gestión profesional de las emociones sentidas no solo por los clientes, sino también por los mismos cuidadores.

La intervención del ergonomista ante los cuidadores que trabajan en centros hospitalarios con cuidado a largo plazo tiene por objetivo, entre otros, documentar las estrategias emocionales experimentadas por los cuidadores. Tiene por otro objetivo informar a los dirigentes, sindicatos y empleados de estos establecimientos en la implantación de modelos de organización de servicio y de cuidado más eficiente. El enfoque "protésico" constituye un ejemplo. Este enfoque da una nueva definición al trabajo prescrito del cuidador. Tiene por objetivo una mejor satisfacción de las necesidades fundamentales de la clientela demente. 
INDEX

Mots-clés : activités de soins, soins aux personnes démentes, ergonomie, agression, stratégies de diversion

Palabras claves : actividades de cuidado, cuidado a las personas dementes, ergonomía, agresión, estrategias de diversión

Keywords : care activities, care for dementia patients, ergonomics, aggressive, diversion strategies

\section{AUTEUR}

\section{MICHEL BIGAOUETTE}

Association pour la santé et la sécurité du travail secteur affaires sociales, 5100 rue Sherbrooke Est, bureau 950, Montréal (Québec), H1V 3R9, Canada, mbigaoue@asstsas.qc.ca 